\title{
Discoidin Domain Receptor 2 Expression as Worse Prognostic Marker in Invasive Breast Cancer
}

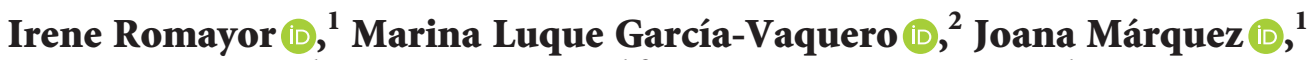 \\ Beatriz Arteta $\left(\mathbb{D},{ }^{1}\right.$ Ramón Barceló, ${ }^{1,3}$ and Aitor Benedicto ${ }^{1}{ }^{1}$ \\ ${ }^{1}$ Department of Cell Biology and Histology, School of Medicine and Nursing, University of the Basque Country (UPV/EHU), \\ 48940 Leioa, Spain \\ ${ }^{2}$ Department of Medicine and Cytometry General Service-Nucleus, CIBERONC, \\ Cancer Research Centre (IBMCC/CSIC/USAL/IBSAL), 37007 Salamanca9, Spain \\ ${ }^{3}$ Oncology Service, Basurto University Hospital, 48002 Bilbao, Spain
}

Correspondence should be addressed to Irene Romayor; irene.romayor@gmail.com

Received 25 October 2021; Accepted 10 February 2022; Published 7 March 2022

Academic Editor: Pranshu Sahgal

Copyright ( 2022 Irene Romayor et al. This is an open access article distributed under the Creative Commons Attribution License, which permits unrestricted use, distribution, and reproduction in any medium, provided the original work is properly cited.

\begin{abstract}
Discoidin domain receptor 2 (DDR2) is arising as a promising therapeutic target in breast carcinoma (BC). The ability of DDR2 to bind to collagen promotes protumoral responses in cancer cells that influence the tumor microenvironment (TME). Nonetheless, the interrelation between DDR2 expression and TME modulation during BC progression remains poorly known. For this reason, we aim to evaluate the correlation between intratumoral expression of DDR2 and the infiltration of the main TME cell populations, cancer-associated fibroblasts (CAFs), and tumor-associated macrophages (TAMs). First, collagen and DDR2 expression levels were analyzed in human invasive $\mathrm{BC}$ samples. Then, $D D R 2$ status correlation with tumor aggressiveness and patient survival were retrieved from different databases. Subsequently, the main pathways, cell types, and tissues correlated with DDR 2 expression in BC were obtained through bioinformatics approach. Finally, we studied the association of DDR2 expression with the recruitment of CAFs and TAMs. Our findings showed that, together with the expected overexpression of TME markers, DDR2 was upregulated in tumor samples. Besides, we uncovered that altered TME markers were linked to DDR2 expression in invasive BC patients. Consequently, DDR2 modulates the stromal reaction through CAFs and TAMs infiltration and could be used as a potential worse prognostic factor in the treatment response of invasive BC.
\end{abstract}

\section{Introduction}

Breast carcinoma (BC) is the most prevalent malignancy in women and the leading cause of death among female population $[1,2]$. Approximately $80 \%$ of all BC cases diagnosed correspond to invasive ductal carcinoma (IDC). Invasive lobular carcinoma (ILC) represents the second most common subclass of BC, with an incidence of around $15 \%$ [3]. Both subtypes differ in clinical, pathological, and biological aspects, with the expression of E-cadherin being the main hallmark of IDC [4]. Apart from its histology, BC tumor is classified according to its molecular phenotype regarding the presence of three different biomarkers: estrogen receptor $(E R)$, progesterone receptor $(P R)$, and human epidermal growth factor receptor 2 (HER2). Thus, luminal-like $\mathrm{BC}$ is characterized by $E R / P R$ positive and HER2 negative, HER2-enriched is defined by ER/PR negative or positive and HER2 positive, and triple-negative tumor is described by the absence of these markers [5]. Current adjuvant treatments are based on targeting these proteins before or after surgery [6, 7], excepting triple-negative BC, for which chemotherapy in combination with immunotherapy regimens are commonly used [8]. However, despite medical advances, more than $20 \%$ of women with early-stage BC will suffer metastatic disease [9], which reflects the need for new targeted therapies.

One of the risk factors involved in BC malignancy is the dysregulation of extracellular matrix (ECM) composition 
and architecture, which is associated with the menopausal status [10] and predisposes women to develop invasive BC later on [11]. Aberrant ECM remodeling is characterized by a progressive collagen enrichment or desmoplasia, which increases mammary gland stiffness and alters the tissue mechanosignaling [12]. Collagen deposition and crosslink provide biochemical and biophysical signals to enhance diverse protumoral characteristics, regarding proliferation, migration, and invasion [13]. Several proteins, such as integrins and specific tyrosine kinase receptors (RTKs), mediate the crosstalk between cancer cells and ECM components [14]. One of the receptors involved in the tumorECM interplay is the discoidin domain receptor 2 (DDR2), an atypical RTK, due to its capacity to bind collagens [15].

$D D R 2$ overexpression has been linked to progression of different cancer types [16], including BC [17-19]. The role of $D D R 2$ in BC development has been uncovered recently, with special emphasis on its ability to stimulate the secretion of collagen-dependent proteases by tumor cells in postpartumrelated carcinomas, activating their migratory and invasive potentials, as well as triggering metastasis [20]. Interestingly, apart from its implication on cancer cells response, DDR2 receptor also mediates key $\mathrm{BC}$ cells' functional features that influence the phenotype and reaction of stromal cells, particularly cancer-associated fibroblasts (CAFs) [21, 22]. CAFs conform to the major component of the cellular fraction of the tumor microenvironment (TME) and actively participate in the desmoplastic process as major producers of extracellular collagen [23]. CAFs increase cancer cells growth through various mechanisms, including proliferation, migration, cytokine and chemokine secretion, and resistance to anticancer drugs $[24,25]$. As well as CAFs, tumor-associated macrophages (TAMs) are a very relevant stromal population during ECM reorganization and carcinogenesis, due to the secretion of several cytokines and chemokines that create a favorable microenvironment for tumor growth, promoting cancer cells proliferation, migration, and chemoresistance [26]. Although CAFs and TAMs communication with tumor cells is essential for BC progression, the role of $D D R 2$ during this interaction remains unclear.

Given the importance of $D D R 2$ in invasive BC process, we evaluated collagen and DDR2 status in tumor samples compared to healthy adjacent tissue, together with DDR2 implication on metastasis progression. Besides, we studied the association of DDR2 expression level and menopausal status with patient survival. Moreover, based on the capacity of DDR2 to modulate the TME, we aimed to analyze the relation between $D D R 2$ expression and the recruitment of CAFs and TAMs into the breast tumors. Our findings revealed that collagen and DDR2 expression levels were significantly higher in BC compared to normal adjacent tissue. In addition, $D D R 2$ upregulation was detected in metastatic tumors and represented a worse prognostic factor in combination with postmenopausal age. Moreover, DDR2 overexpression correlated with a higher CAFs and TAMs infiltration into the mammary tumor tissue. Taking these results together, $D D R 2$ could play an important role in the modulation of the TME and may represent a potential target for antitumor early and metastatic BC treatments.

\section{Materials and Methods}

2.1. Patients and Tissue Samples. Breast IDC samples and the corresponding adjacent nontumoral or healthy tissues were obtained by mammary resections from 25 patients $(n=25)$. Adjacent nontumoral samples were collected from regions at least $5 \mathrm{~mm}$ away from tumor boundaries and were classified as normal breast tissue by a histopathologist. Although histological normalcy was detected, we must consider that carcinogenic alterations could affect the phenotypic and genetic profiles of the tissue regions up to $1 \mathrm{~cm}$ from the tumor margins [27]. Additionally, clinical and pathological features including menopausal status, tumor size, histological grade, tumor stage, proliferative index, e-cadherin expression, hormonal receptors-HER2/neu status, and lymph node invasion were recorded from the medical history (Table 1). Tumor staging was established according to the International Union against Cancer Tumour-NodeMetastasis (UICC-TNM) classification. All samples were provided by Basurto University Hospital Biobank (Basque Country, Spain) under the guidelines of the Institutional Ethics Committee with the corresponding informed consent.

2.2. Masson's Trichrome Staining. The detection of collagen type I fibers was performed through Masson's trichrome staining. In brief, $5 \mu \mathrm{m}$ tissue slides were incubated with Weigert's iron hematoxylin solution for 10 minutes and Biebrich scarlet-acid fuchsin Solution for 15 minutes. Finally, samples were treated with phosphomolybdic acid solution for 15 minutes and aniline blue solution for 5 minutes. All staining solutions were purchased from SigmaAldrich, United States (cat. numbers HT1079, HT151, HT153, and B8563, respectively). Collagen fibers were observed in blue, and expression level was quantified using FIJI-ImageJ (Colour Deconvolution plugin).

2.3. Immunohistochemistry and Scoring. The expressions of DDR2, $\alpha$-SMA, and CD68 proteins were analyzed in consecutive sections of breast IDC and nontumoral samples. $5 \mu \mathrm{m}$ tissue slides were pretreated with Antigen Retrieval Solution (R\&D Systems, United States, cat. number CTS013) for 10 minutes at $90^{\circ} \mathrm{C}$. Endogenous peroxidase activity was blocked using 3\% hydrogen peroxide (PanReac AppliChem, United States, cat. number 131077) in phosphate-buffered saline (PBS) 1X for 30 minutes at room temperature. Then, slides were incubated with $0.4 \%$ Triton X-100 (Thermo Fisher Scientific, United States, cat. number 85112) and 5\% fetal bovine serum (FBS, Thermo Fisher Scientific, cat. number 10091148) in PBS $1 \mathrm{X}$ for 1 hour at room temperature and overnight at $4^{\circ} \mathrm{C}$ with one of the following primary antibodies: anti-DDR2 1:500 (GeneTex, United States, cat. number GTX25520), anti- $\alpha$-SMA $1: 200$ (Dako, United States, cat. number GA61161-2), and antiCD68 1:250 (Thermo Fisher Scientific, cat. number MA513324). Antibodies were detected with either biotinylated anti- 
TABLE 1: Clinicopathologic characteristics of BC patients $(n=25)$.

\begin{tabular}{|c|c|}
\hline Characteristics & $N(\%)$ \\
\hline \multicolumn{2}{|l|}{ Menopausal status (median age $=63,8$ years) } \\
\hline Premenopause & $6(24)$ \\
\hline Postmenopause & $19(76)$ \\
\hline \multicolumn{2}{|l|}{ Tumor size $(\mathrm{cm})$} \\
\hline$\leq 2.5$ & $13(52)$ \\
\hline$>2.5$ & $10(40)$ \\
\hline Unknown & $2(8)$ \\
\hline \multicolumn{2}{|l|}{ Tumor grade } \\
\hline I & $0(0)$ \\
\hline II & $10(40)$ \\
\hline III & $15(60)$ \\
\hline \multicolumn{2}{|l|}{ T stage } \\
\hline T0 & $1(4)$ \\
\hline $\mathrm{T} 1$ & $4(16)$ \\
\hline $\mathrm{T} 2$ & $20(80)$ \\
\hline \multicolumn{2}{|l|}{ N Stage } \\
\hline No & $10(40)$ \\
\hline N1 & $9(36)$ \\
\hline N2 & $5(20)$ \\
\hline N3 & $1(4)$ \\
\hline \multicolumn{2}{|l|}{ M stage } \\
\hline M0 & $25(100)$ \\
\hline \multicolumn{2}{|l|}{ Proliferative index (Ki-67 staining) } \\
\hline Low $(<20 \%)$ & $3(12)$ \\
\hline High $(\geq 20 \%)$ & $22(88)$ \\
\hline \multicolumn{2}{|l|}{ E-cadherin } \\
\hline Positive (IDC subtype) & $25(100)$ \\
\hline Negative (ILC subtype) & $0(0)$ \\
\hline \multicolumn{2}{|l|}{ Hormonal receptors-HER2/neu status } \\
\hline$E R / P R(+)-H E R 2(-)$ (luminal-like) & $9(36)$ \\
\hline ER/PR (+)-HER2 (+) (HER2-enriched) & $10(40)$ \\
\hline$E R / P R(-)-H E R 2(+)$ (HER2-enriched) & $2(8)$ \\
\hline$E R / P R(-)-H E R 2(-)$ (triple-negative) & $4(16)$ \\
\hline \multicolumn{2}{|l|}{ Lymphovascular invasion } \\
\hline Present & $15(60)$ \\
\hline Absent & $10(40)$ \\
\hline
\end{tabular}

rabbit 1:1500 or anti-mouse 1:2000 for 1 hour at room temperature, followed by streptavidin-HRP 1:500 for 30 minutes at room temperature and DAB Substrate Kit for 2-3 minutes (all purchased from Thermo Fisher Scientific, cat. numbers 31820, 31800, SA10001, and 34002, respectively). Finally, slides were counterstained in hematoxylin and mounted with Sub-X Mounting Medium (Leica Biosystems, Germany, product ID SUB-X-MOUNTING-MEDIUM). Protein expression was quantified using FIJI-ImageJ (Colour Deconvolution plugin).

The expressions of DDR2, $\alpha$-SMA, and CD68 proteins were evaluated in the whole tissue for intensity of staining comparing breast IDC samples to nontumor ones. Staining with relative values up to 3 -fold increase was classified as low status, while that with values superior to 3 -fold increase was classified as high status. This evaluation was made following the criteria described by Toy et al. [28]. Samples 4, 12, and 23 ( $\alpha$-SMA staining), as well as 23 and 24 (CD68 staining), were excluded from the correlation analysis due to the high percentage of fatty tissue detected, which made it complicated to classify them as low or high status.
2.4. RT-qPCR. Total RNA was extracted and purificated from breast IDC and nontumoral samples (including the stromal compartment) using the Total RNA Purification Kit (Norgen Biotek Corp., Canada, cat. number 17250). RNA concentration and quality were assessed by NanoDrop spectrophotometer (ND-1000; Thermo Fisher Scientific) and $0.5-1 \mu \mathrm{g}$ of RNA was reverse-transcribed into cDNA with iScript ${ }^{\mathrm{TM}}$ Reverse Transcription Supermix (BIO-RAD, United States, cat. number 1708841). Quantification of cDNA template was performed with real-time PCR using iTaq $^{\text {тм }}$ Universal SYBR ${ }^{\circledR}$ Green Supermix (BIO-RAD, cat. number 1725121) in ABI 7900HT (Life Technologies). PCR primers (Life Technologies) were as follows: DDR2 F, GGAGGTCATGGCATCGAGTT, and R, GAGTGCCATCCCGACTGTAATT; GAPDH (housekeeping) F, GTATGACTCCACTCACGGCAA, and R, CTTCCCATTCTCGGCCTTG; RPS15 (housekeeping) F, AGACGAGTTTCAGTGTTGCC, and R, AGACCACAGCCTCAGACAAG. Relative expression of target genes was normalized to the internal control genes GAPDH and $R P S 15$ by the $\triangle \triangle \mathrm{Ct}$ method. Data were generated by the use of specific software (ABI Prism, SDS2.0, Life Technologies) after normalization. The experiments were performed in triplicate.

2.5. Bioinformatic Analysis. For analysis of gene and DDR2 expression correlation with TME markers, normal $(n=15)$ and invasive BC, IDC $(n=1237)$, and ILC $(n=116)$, microarray profiles generated by METABRIC project $[29,30]$ were retrieved from cBioPortal [31, 32]. Besides, DDR2 expression profile based on the Cyclin D1 (CCND1) gene status as metastatic BC marker $[33,34]$ (with CCND1 amplification, $n=12$; without CCND1 amplification, $n=80$ ) and the effect of $D D R 2$ expression level and menopause status on patients survival (high DDR2/perimenopause, $n=9$; high $D D R 2 /$ postmenopause, $n=156$; high $D D R 2 /$ premenopause, $n=75$; low $D D R 2 /$ perimenopause, $n=28$; low DDR2/postmenopause, $n=532$; low $D D R 2 /$ premenopause, $n=152$ ) were obtained from UALCAN Cancer Database (datasets ID: MET500 and The Cancer Genome Atlas, TCGA) [35]. Then, the 100 most coexpressed genes with DDR2 in invasive BC (Table S1) were acquired from Gene Expression Profiling Interactive Analysis Database 2 (GEPIA2; datasets ID: The Cancer Genome Atlas Program, TCGA, and Genotype-Tissue Expression Project, GTEx) [36]. Finally, KEGG 2019 Human and ARCHS4 Tissues platforms were applied to explore the biological functions and tissue correlation of DDR2 during BC by using the Enrichr Database [37].

2.6. Statistical Analysis. Data were analyzed using GraphPad Prism software (version 6) and are presented as the mean \pm standard deviation (SD). Differences in gene and protein expression levels were analyzed by two-tailed Student's $t$-test. The association between DDR2 and stromal markers expression was analyzed by Chi-square, Fisher's exact test, and Pearson's correlation coefficient. The criterion for significance was $p \leq 0.05$ for all comparisons. 


\section{Results}

3.1. Collagen and DDR2 Expression Are Upregulated in $\mathrm{Hu}$ man Invasive BC Compared to Nontumoral Tissue. Collagen accumulation and DDR2 high expression have been reported in invasive $\mathrm{BC}$ both in vitro and in vivo $[17-19,38,39]$. We analyzed the intratumoral levels of collagen type I and DDR2 in 25 mammary tissue samples from IDC (e-cadherin positive) and their matched adjacent normal tissues. As observed in Figure 1, the vast majority of the tumors showed collagen deposition $(p=0.0209)$ and upregulated DDR2 protein $(p<0.0001)$ compared to that in nontumoral tissue. Besides, DDR2 quantification in the RNA samples obtained from IDC patients supported this finding, as demonstrated by RT-qPCR $(p=0.0004)$. Further analysis of DDR2 status using METABRIC Database confirmed that $D D R 2$ mRNA levels were significantly higher in ductal and lobular BC subtypes, with respect to those in the normal breast tissue ( $p=0.0381$ and $p=0.0023$, respectively).

\subsection{DDR2 Upregulation Is Associated with BC Metastatic} Progression and Low Postmenopausal Patient Survival. $D D R 2$ overexpression has been related to poor prognosis in several cancer types, including BC $[18,28]$. As observed in Figure 2, metadata analysis showed that $D D R 2$ gene level was higher in those invasive BC tumors with a more aggressive phenotype $(p=0.0318)$, defined by the CCND1 amplification as metastatic marker [33]. Moreover, regarding patient age, high DDR2 expression notably increased postmenopausal BC patients' mortality, compared to the postmenopausal women who exhibited low DDR2 expression levels $(p=0.03)$. On the contrary, DDR2 expression was not relevant in pre- and perimenopausal women. Thus, DDR2 status was only significant when we analyzed postmenopausal BC patients.

3.3. DDR2 Expression in BC Is Corrlated with Cancer-Associated Genes and Stromal Cells Markers. In order to evaluate the role of DDR2 during BC development, we studied the function of the main genes coexpressed with DDR2 in the mammary tumor tissue. On the one hand, KEGG 2019 Human Database indicated that DDR2 was predominantly related to ECM interplay and cell adhesion, two features directly linked with DDR tyrosine kinase family, along with cancer signaling pathways. On the other hand, ARCHS4 Tissues Database revealed that 4 out of 10 of the most associated cells with DDR2 in BC were from fibroblast origin (fibroblasts, foreskin fibroblasts, and myoblasts), together with macrophages, thus showing a stromal pattern (Figure 2).

\subsection{Enhanced Expression of $\alpha-S M A$ and CD68 in Human} Breast IDC. CAFs and TAMs are active players in BC progression and chemoresistance $[25,26]$. We evaluated the infiltration of CAFs and TAMs in breast IDC samples by $\alpha-S M A$ and $C D 68$ staining quantification, respectively. In the healthy breast tissue, these markers correspond to fibroblast-like cells and macrophages, which maintain mammary gland homeostasis. Thus, the comparison of $\alpha-S M A$ and CD68 expression levels between normal and tumor samples represents the mobilization of these stromal cell populations during the tumorigenic process. As observed in Figure 3, we found increased expressions of $\alpha$-SMA and CD68 markers in the peritumoral and intratumoral areas compared to normal adjacent tissue. These results revealed a pronounced recruitment of CAFs and TAMs in the developing tumoral tissue from breast IDC patients.

3.5. Overexpression of DDR2 Correlates with Increased CAFs Infiltration in Human Invasive BC. The TME is a dynamic compartment that drives tumor growth. CAFs are a major player in this ecosystem, supporting BC growth through several pathways [25]. We aimed to elucidate whether the intratumoral DDR2 expression may be related to the recruitment of CAFs and, therefore, could be used as a marker of stromal reorganization during BC. Using Chisquare and Fisher's exact test, we found a strong correlation between intratumoral $D D R 2$ protein levels and CAFs infiltration (characterized by $\alpha$-SMA expression) in breast IDC samples. As observed in Figure 4, low DDR2 expression was directly linked with reduced CAFs recruitment $(p=0.0008)$. In depth, $75 \%$ of the low DDR2 expressing tumors exhibited low $\alpha$-SMA staining, while CAFs infiltration was high in only $25 \%$ of the low DDR2 levels breast tumors. On the other hand, $64 \%$ of the high DDR2 expressing tumors showed elevated $\alpha$-SMA protein levels, while barely $36 \%$ of these tumors presented low $\alpha-S M A$ expression. Additional analysis of DDR2 and $\alpha$-SMA correlation in IDC and ILC using METABRIC Database supported this finding $(p<2.2 e-16$ and $p<1.9 e-08$, respectively), uncovering a direct relation between tumoral DDR2 expression and CAFs recruitment.

3.6. TAMs Recruitment Correlates with DDR2 Upregulation in Human Invasive BC. TAMs, key immunomodulatory cells, are a significant source of cytokines and chemokines that promote disease progression [26]. Here we analyzed the correlation of infiltrating CD68 positive macrophages and the expression of intratumoral $D D R 2$ in breast IDC samples. Chi-square and Fisher's exact test revealed a deep correlation between high intratumoral DDR2 expression and elevated infiltration of CD68 positive TAMs $(p=0.0001)$. Strikingly, low macrophages counts were found only in patients with low levels of intratumoral DDR2. However, $67 \%$ of high DDR2 expressing tumors showed elevated macrophages recruitment, and only $33 \%$ of these tumors presented low CD68 positive cells counts (Figure 5). Further analysis of DDR2 and CD68 correlation in IDC and ILC using METABRIC Database confirmed our results $(p<2.2 e-16$ and $p<3.3 e-07$, respectively), suggesting a feasible link between intratumoral DDR2 expression and TAMs infiltration. 


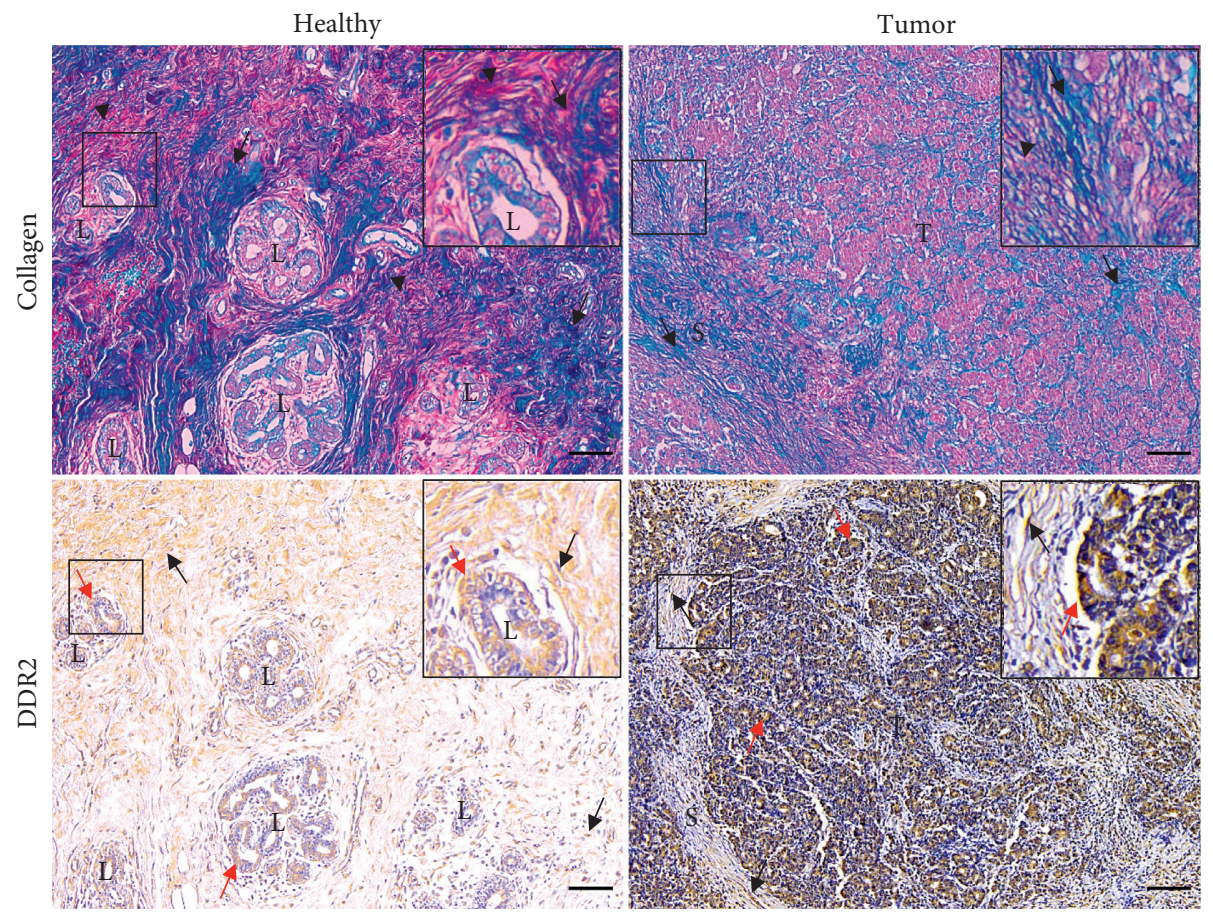

(a)

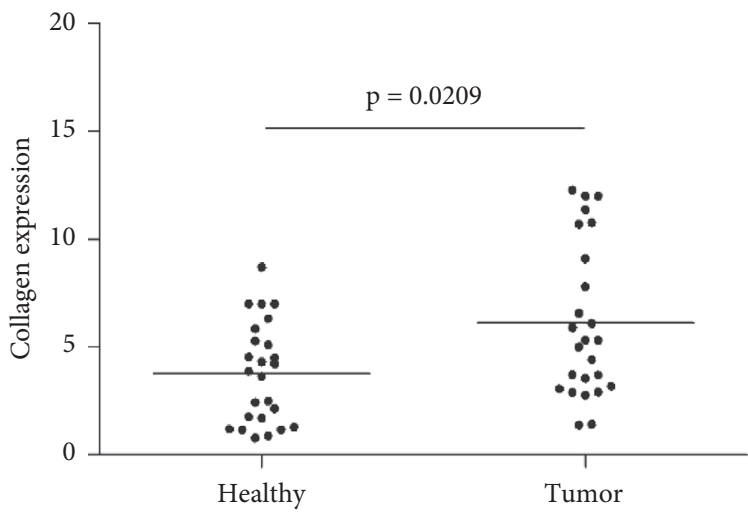

(b)

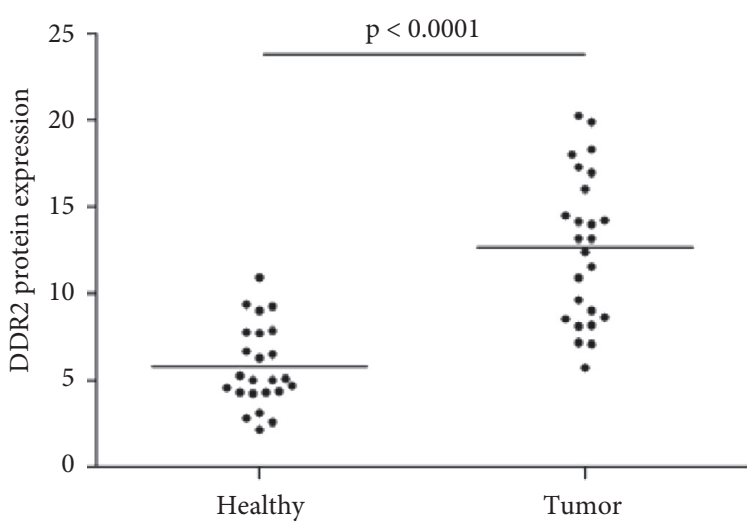

(c)

FIgUre 1: Continued. 


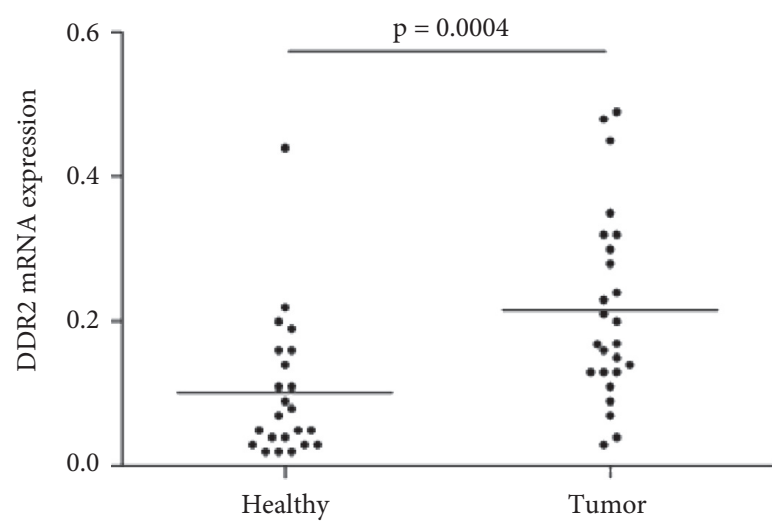

(d)

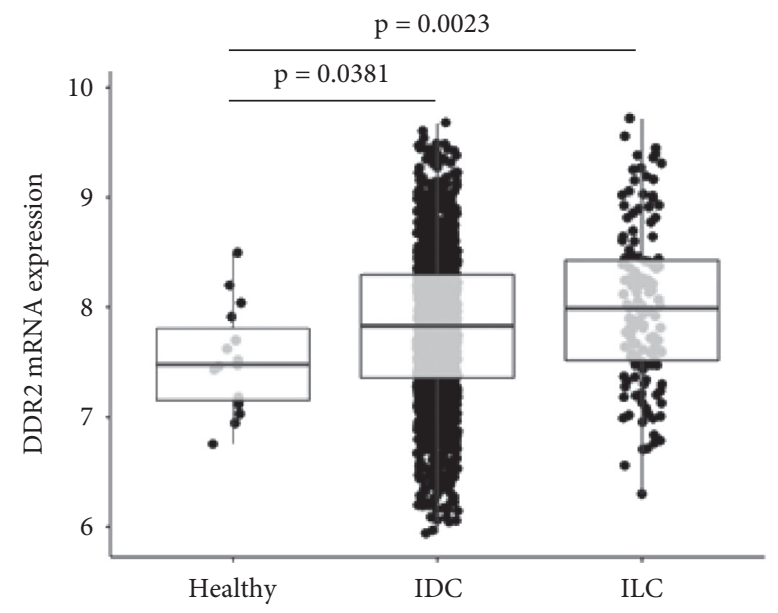

(e)

FIGURE 1: Expression of collagen and DDR2 in human invasive BC. Masson's trichrome staining shows noncollagenous fibers in red (black arrow heads) and collagen type I fibers in blue (black arrows). Combination of both fibers appears in healthy breast tissue, while tumor tissue predominantly exhibits collagen expression. Immunohistochemistry shows that healthy mammary gland epithelial cells and tumor cells express DDR2 (red arrows). Besides, DDR2 staining is observed in the stroma of normal and tumor samples (black arrows). L: lobule, T: tumor, S: stroma. Scale bar: $100 \mu \mathrm{m}$. Insets: $2 x$ magnification (a). Staining quantification (colored area per total tissue area) indicates that collagen $(p=0.0209)$ and $D D R 2(p<0.0001)$ expressions are significantly higher in breast IDC samples (b, c). RT-qPCR demonstrates that relative expression of $D D R 2$ is significantly upregulated in breast cancerous tissues when compared to that in nontumoral tissues (including the stromal compartment) $(p=0.0004)$ (d). Mean mRNA levels of DDR2 are overexpressed in breast IDC $(p=0.038)$ and ILC $(p=0.0023)$ samples obtained from METABRIC BC Datasets (e). Data are expressed as the mean $\pm \mathrm{SD}$.

\section{Discussion}

The ECM is one of the main components of the TME. The importance of these extracellular elements resides in their ability to promote significant changes in the behavior of cancer cells through receptor-mediated ECM-tumor cell interactions. These alterations exert a positive effect on cancer cells, by means of chemoresistance, as well as increased proliferation and migration, among others [40]. Regarding BC, the first most prevalent malignant disease among women, collagen accumulation has been postulated as a risk factor $[38,41]$. Despite the limited number of patients, and taking into account the fact that the heterogeneity of the BC population represents a limiting factor, the analyzed invasive BC tissue samples exhibit a marked collagen deposition, pointing out the crosstalk between ECM and $\mathrm{BC}$ cells as a feasible starting point for tumor development. Even though collagen constitutes an integral and functional key player of cancer tissue regulation, current therapies against the variety of histological and molecular BC subtypes do not target cellular components directly linked with this ECM protein.

The collagen-binding RTK $D D R 2$ is known to play important roles in tumor progression [16]. In the mammary carcinogenic tissue, DDR2 expression has been postulated as an independent prognostic value [18], with a critical role during postpartum-associated BC $[20,42]$. In this regard, increased DDR2 promotes tumor aggressiveness in the breast, by means of enhanced proliferation, migration, colony formation, and metastasis [17, 19]. In this work, we show that DDR2 expression is upregulated in a small sample size composed by different invasive $\mathrm{BC}$ molecular profiling cohorts, including luminal-like, HER2-enriched, and triplenegative subclasses. Besides, the later bioinformatics analysis 


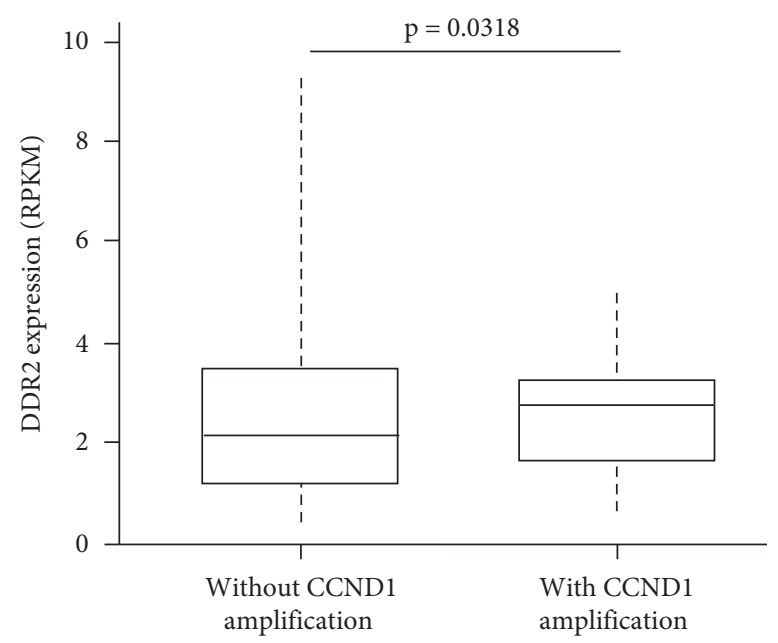

(a)

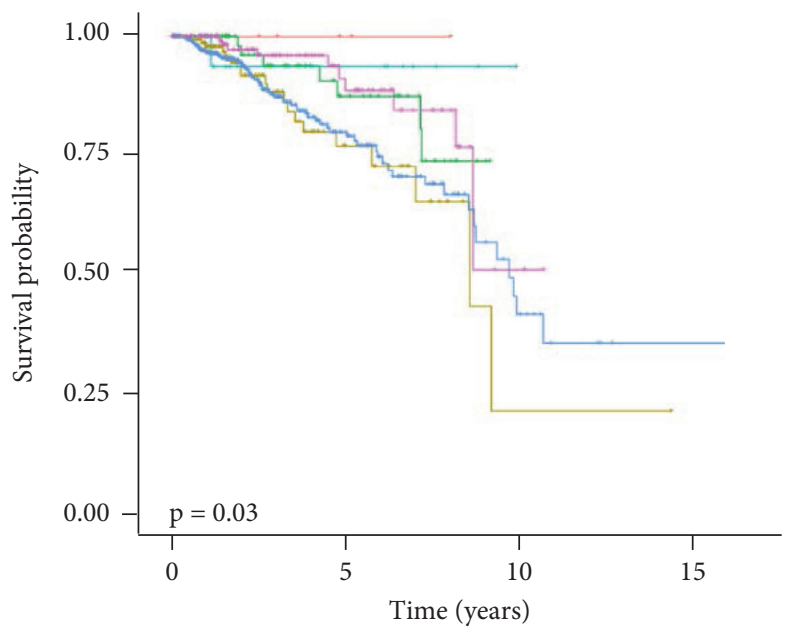

- High DDR2/Peri-menopause $(\mathrm{n}=9)$

- High DDR2/Post-menopause $(\mathrm{n}=156)$

High DDR2/Pre-menopause $(\mathrm{n}=75)$

Low DDR2/Peri-menopause $(\mathrm{n}=28)$

- Low DDR2/Post-menopause $(\mathrm{n}=532$

— Low DDR2/Pre-menopause $(\mathrm{n}=152)$

(b)

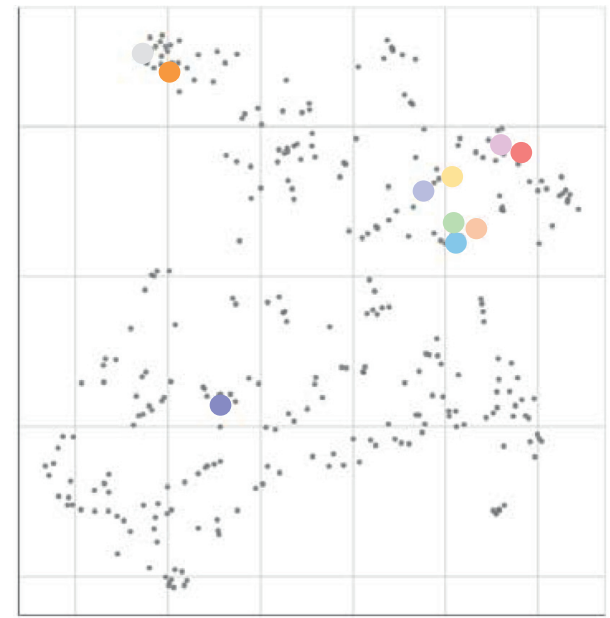

ECM-receptor interaction 7.63e-04

Focal adhesion $3.25 \mathrm{e}-03$

Human papillomavirus infection $6.28 \mathrm{e}-03$

Small cell lung cancer $1.14 \mathrm{e}-02$

Prostate cancer $1.27 \mathrm{e}-02$

Axon guidance $1.3 \mathrm{e}-02$

MicroRNAs in cancer $1.72 \mathrm{e}-02$

Toxoplasmosis $1.91 \mathrm{e}-02$

Glycosaminoglycan biosynthesis $2.89 \mathrm{e}-02$

Cell adhesion molecules $3.68 \mathrm{e}-02$

\begin{tabular}{llll}
\hline 0 & 2 & 2 & 3
\end{tabular}

(c)

Figure 2: Continued. 

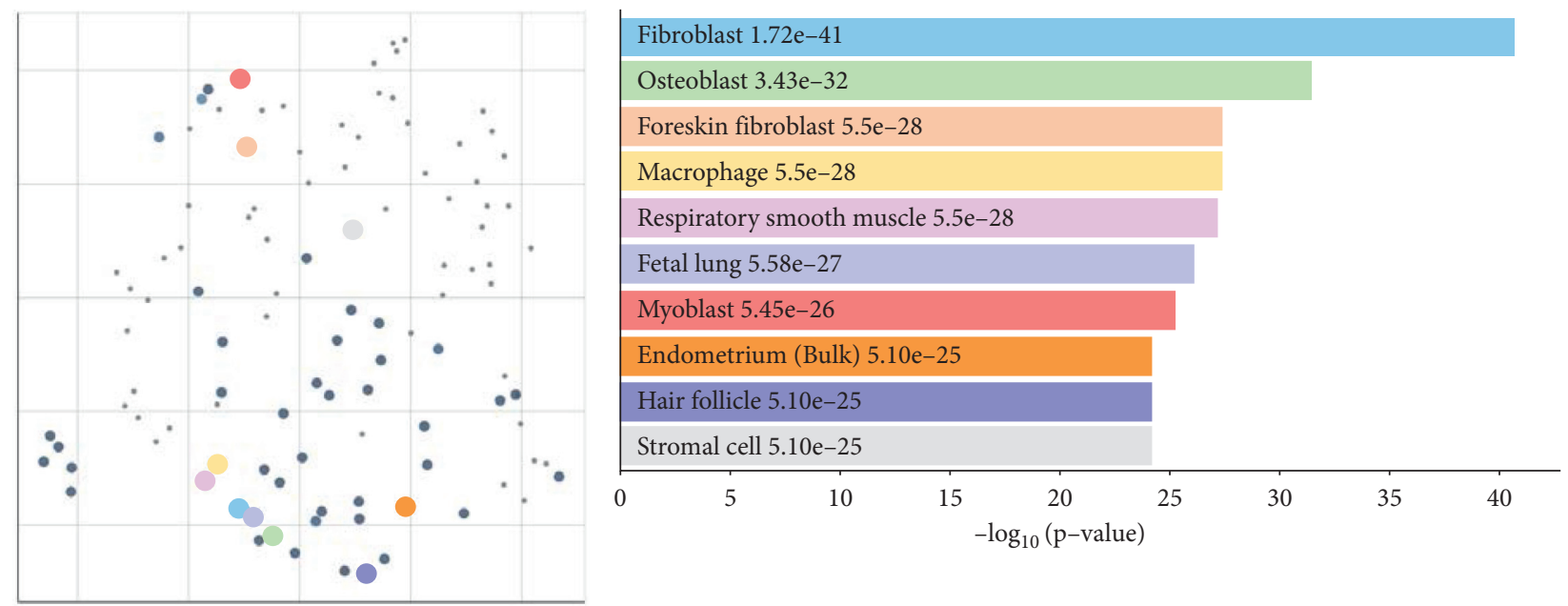

(d)

FIGURE 2: Implication of DDR2 expression on invasive BC progression, patients' outcome, and cell signaling pathways. UALCAN Cancer Database reveals that mean mRNA levels of DDR2 are upregulated in breast tumor samples with CCND1 gene amplification compared to those without CCND1 overexpression ( $p=0.0318$ ). RPKM: reads per kilobase of transcript (a). Moreover, concerning menopausal age (pre, peri-, or postmenopause women), high DDR2 expression in combination with postmenopausal status significantly decreases patient survival compared to low DDR2 status $(p=0.03)$. The comparison between the other groups does not show significant results $(p>0.05)(b)$. KEGG 2019 Human pathways (c). and ARCHS4 Tissues expression (d). correlation with DDR2 in invasive BC. Scatter plots (left) represent gene clusters according to their similarity on a map. Colored circles correspond to the Top 10 enriched terms visualized in the bar chart together with their $p$ values (right).

including a large number of patients confirms these results. As for the last, Toy et al. [28] described that high DDR2 protein levels were significantly associated with poor triplenegative BC patients' outcome, evidencing the involvement of $D D R 2$ in tumor malignancy. In this line, we detect DDR2 overexpression in metastatic $\mathrm{BC}$ patients with CCND1 gene amplification, a worse survival predictive biomarker in breast tumors [33, 34]. Furthermore, we uncover that mortality increase in those women with a high DDR2 expression status along with postmenopausal age. Thus, elevated expression of DDR2 could reflect a worse prognosis in those postmenopausal BC patients. Taken together, these results suggest that DDR2 may participate in tumor cells growth and permanence. In fact, bioinformatics analyses corroborate that $D D R 2$ signaling in $\mathrm{BC}$ is widely connected to cancer-associated pathways, apart from those related to ECM interaction. Interestingly, the examination of the $D D R 2$ expression pattern indicates that this receptor represents an important part of the stromal compartment, mostly detected in fibroblasts and macrophages. This goes in line with the immunohistochemical analysis, in which we observe DDR2 expression in the tumor stroma of $\mathrm{BC}$ tissue slides.

The TME is known to support cancer progression, influencing therapeutic response and clinical outcome. Thus, infiltrating CAFs and TAMs, the main TME cell populations, maintain cancer development [43]. Regarding stromal cells markers detection in 25 BC patients, $\alpha-S M A$ and $C D 68$ upregulation reveals a high recruitment of these cells into the mammary tumor tissue. Concerning $\alpha-S M A$ detection, it is also found in vascular muscular cells and pericytes, which account in high densities in tumor tissues
[44]. Even though the heterogeneity of CAFs in the TME cannot be described using a single marker, $\alpha$-SMA expression has been extensively analyzed as one of the main myofibroblast markers of the tumor stroma $[45,46]$. Intriguingly, adding our small number of samples with the extensive datasets available, we observe a correlation between DDR2 overexpression and increased levels of CAFs and TAMs in the malignant tissue. This finding suggests that the stromal fraction of $\mathrm{BC}$ may be modulated by tumor $D D R 2$. Aside from cancer cell intrinsic effects, $D D R 2$ is also involved in several processes that alter the breast TME, facilitating disease progression. This could be controlled by the wide spectrum of DDR2 mediated changes in the tumor stroma. In this line, Corsa et al. proposed that DDR2 expressed by BC cells determines CAFs activation to enhance tumor invasion and metastasis [22]. Similarly, tumor $D D R 2$ regulates collagen signaling in CAFs during $\mathrm{BC}$ progression, as recently reported by Bayer et al. [21]. Comparably, we uncover a plausible positive interrelation between the levels of DDR2 and the mobilization of CAFs and TAMs into the tumor foci, which points out to DDR2 as a possible regulator of attracting signals for these cell populations. Concerning the latter, it is well established that DDR2 is implicated in metalloproteinases (MMPs) secretion by tumor cells [47], resulting in ECM degradation and facilitating stromal cells infiltration and cancer cells migration and invasion [48]. Thus, MMPs production by BC cells through DDR2 modulation could promote CAFs and TAMs recruitment. Eventually, although CAFs and TAMs are defined as cancer progression promotors, these stromal cells can also delay tumor growth, which depends on the subtype of CAFs and TAMs populations [49-52]. Considering its 


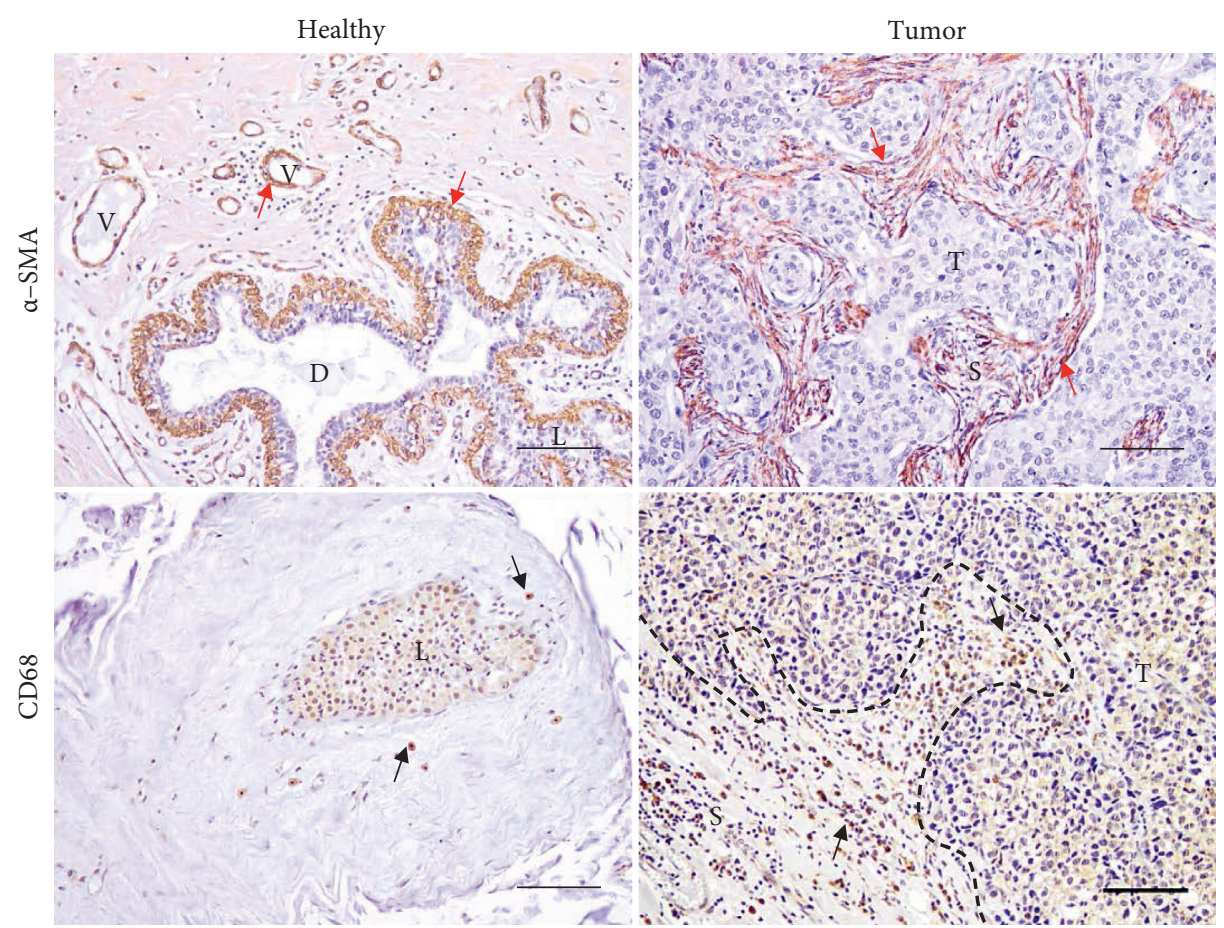

(a)

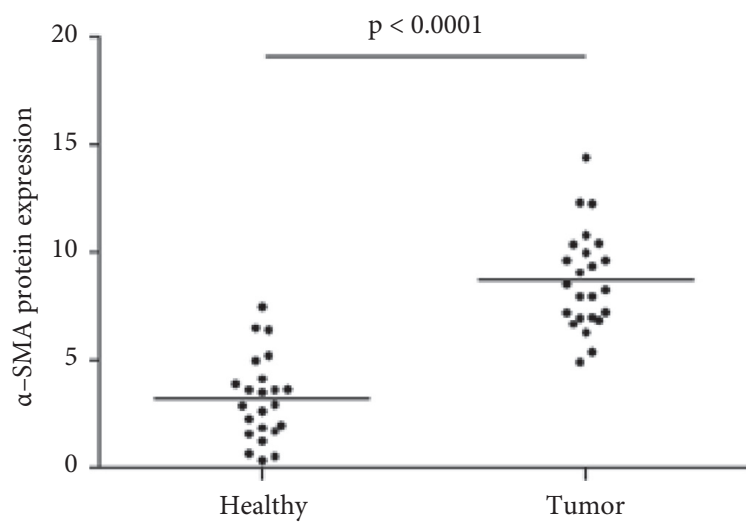

(b)

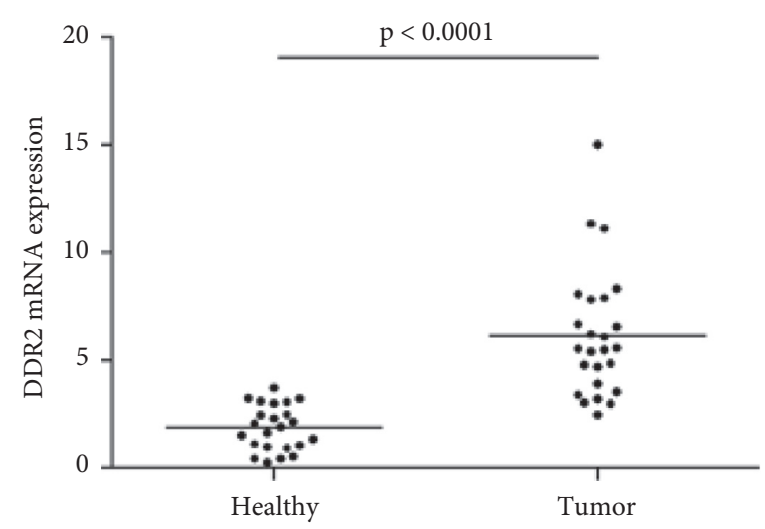

(c)

Figure 3: Expression of $\alpha$-SMA and CD68 in nontumoral breast tissue and breast IDC samples. Immunohistochemistry shows that normal myoepithelial cells and vessels (V) express $\alpha$-SMA. Stromal fibroblasts from tumor samples also express $\alpha$-SMA (red arrows). Stromal macrophages from normal and tumor tissues express CD68 (black arrows). D: duct; L: lobule; T: tumor, S: stroma. Scale bar: $50 \mu \mathrm{m}$ (a). Staining quantification (colored area per total tissue area) shows that stromal cells markers expression is significantly upregulated in breast IDC samples $(p<0.0001)(b, c)$. Data are expressed as the mean \pm SD.

dual role, additional analysis evaluating CAFs and TAMs subpopulations markers may elucidate their specific phenotype.

Other DDR2 stimulated proteins are TGF- $\beta$ and parathyroid hormone-related protein $(P T H r P)$, known to play a role in bone metastasis [53]. On the one hand, TGF- $\beta$ contributes to CAFs infiltration and proliferation, which produce excessive ECM deposition, providing a scaffold for the entrance of immune cells, such as TAMs, and a substrate for cell migration $[54,55]$. On the other hand, PTHrP induces the epithelial-to-mesenchymal transition (EMT), by which epithelial cells transform in fibroblasts in several tissues [56, 57, 58]. This process may account for breast epithelium, therefore consisting in a fibroblast source related to DDR2. Interestingly, in bone metastasis, $\mathrm{PTHrP}$ seems to stimulate the recruitment of macrophages into the tumor promoting the secretion of CCL2 by osteoblasts [56]. It is tempting to hypothesize that a similar process may occur in breast tissue, driving to TAMs accumulation.

This work represents one of the few preliminary studies correlating DDR2 expression with CAFs and TAMs recruitment during invasive $\mathrm{BC}$ progression. However, further ongoing studies will expand the relation between these markers during $\mathrm{BC}$ development. Our prior conclusions reflect that DDR2 may constitute an alternative therapeutic target, especially for those individuals with invasive $\mathrm{BC}$ or 


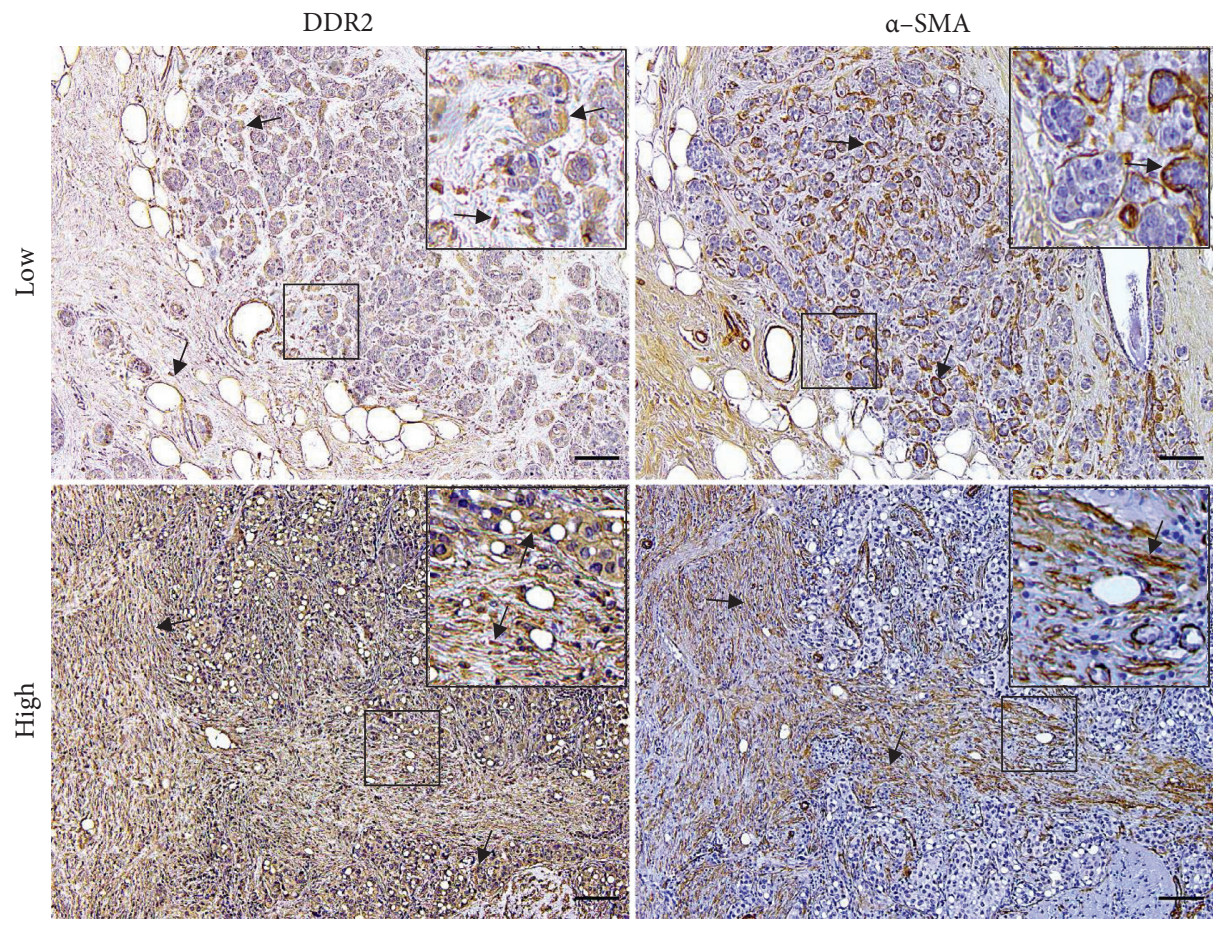

(a)

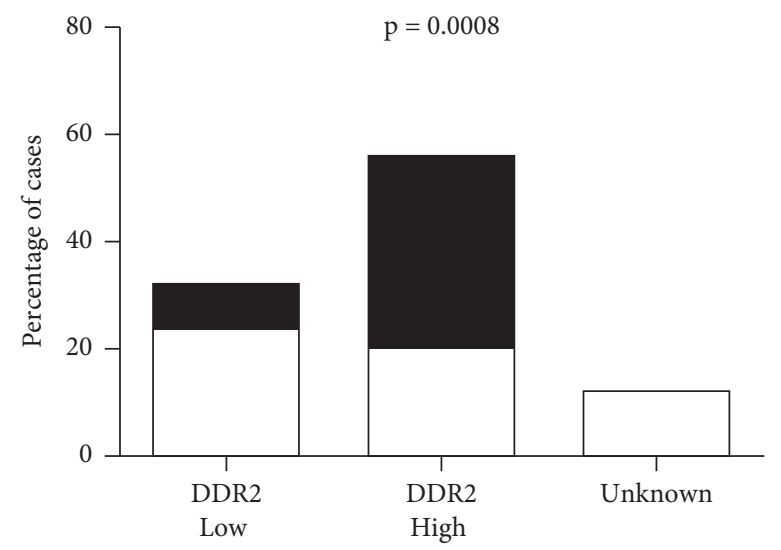

\begin{tabular}{|c|c|c|}
\hline Status & $\alpha$-SMA Low & $\alpha$-SMA High \\
\hline DDR2 Low & $6(75 \%)$ & $2(25 \%)$ \\
\hline DDR2 High & $5(36 \%)$ & $9(64 \%)$ \\
\hline
\end{tabular}

$\square$ a-SMA Low

(b)

Figure 4: Continued. 


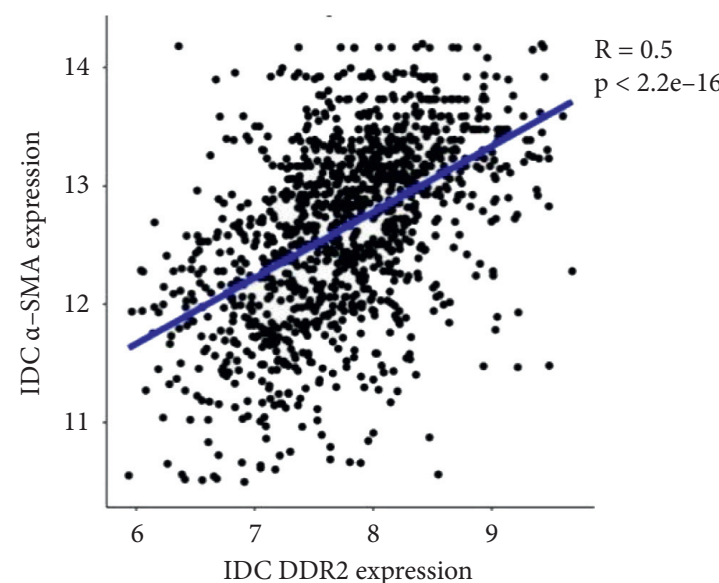

(c)

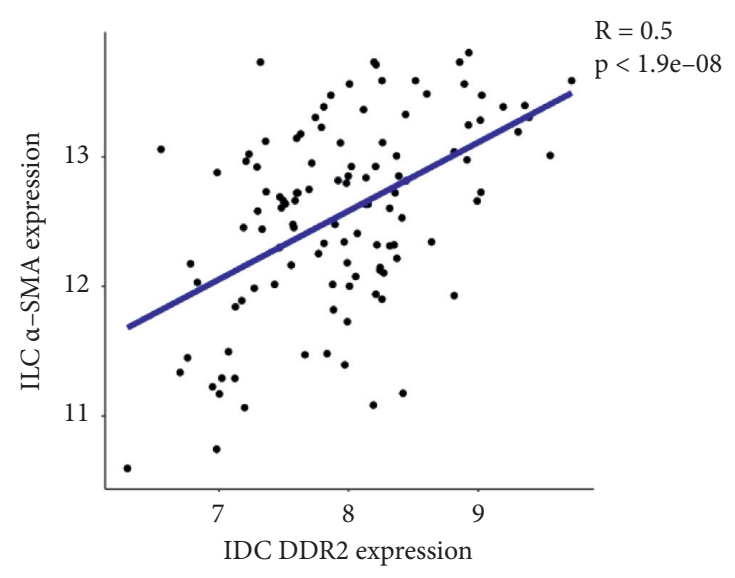

(d)

FIGURE 4: Interplay between the expressions of $D D R 2$ and $\alpha$-SMA in human invasive BC. Images represent characteristic immunohistochemical samples of DDR2 and $\alpha$-SMA low and high expression (arrows). Scale bar: $100 \mu \mathrm{m}$. Insets: 2x magnification (a). Graph shows that $D D R 2$ expression is related to $\alpha$-SMA levels $(p=0.0008)(\mathrm{b}) . D D R 2$ and $\alpha-S M A$ status positive correlation in breast IDC ( $R=0.5$; $p<2.2 e-16)$ and ILC $(R=0.5 ; p<1.9 e-08)$ samples obtained from METABRIC BC Datasets $(\mathrm{c}, \mathrm{d})$.

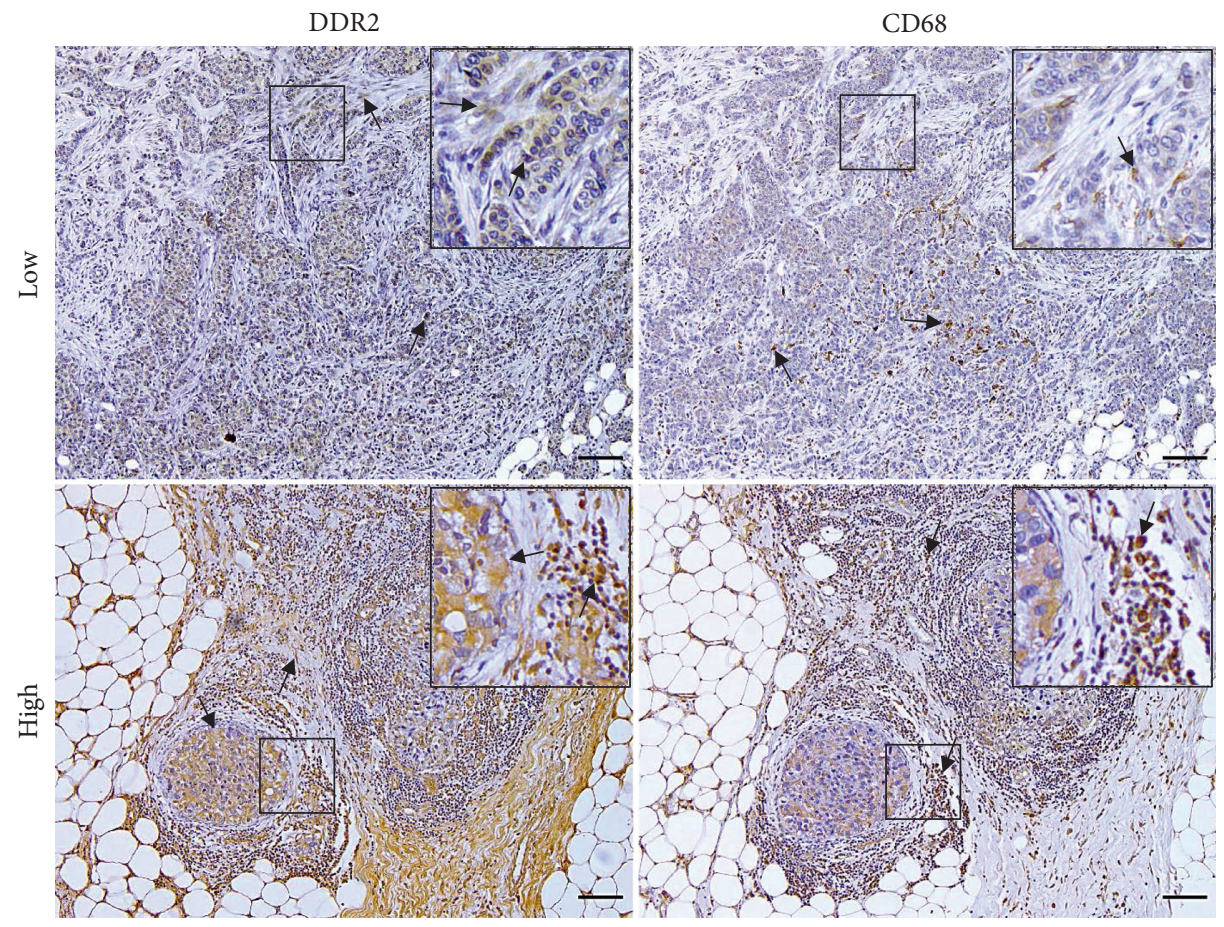

(a)

Figure 5: Continued. 


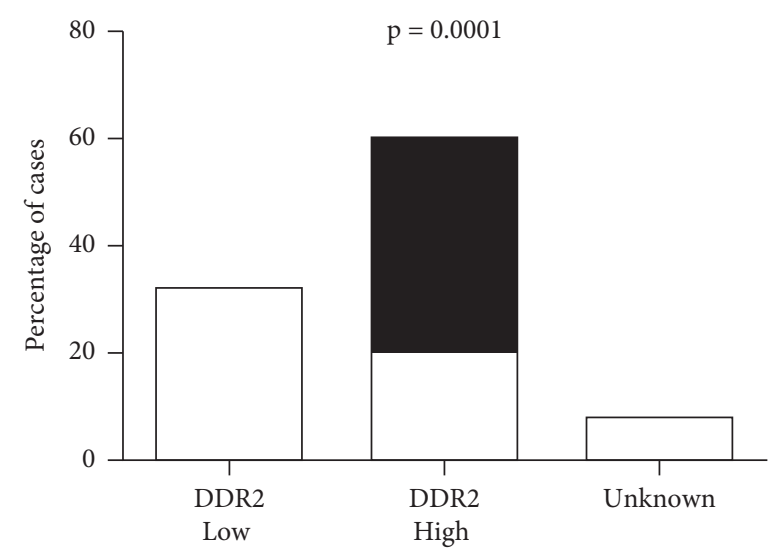

\begin{tabular}{|c|c|c|}
\hline Status & CD68 Low & CD68 High \\
\hline DDR2 Low & $8(100 \%)$ & 0 \\
\hline DDR2 High & $5(33 \%)$ & $10(67 \%)$ \\
\hline
\end{tabular}

$\square$ CD68 Low

- CD68 High

(b)

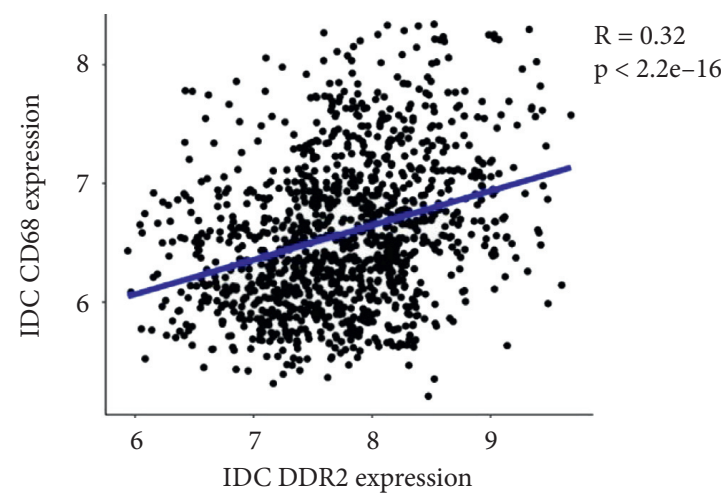

(c)

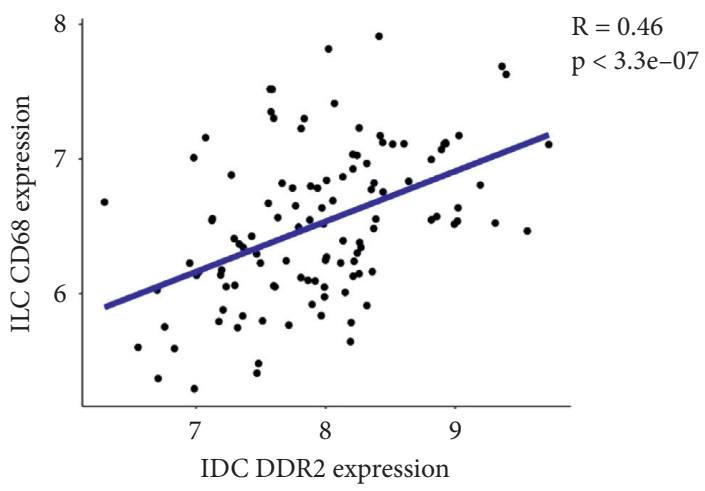

(d)

FIGURE 5: Interplay between the expressions of DDR2 and CD68 in human invasive BC. Images represent characteristic immunohistochemical samples of DDR2 and CD68 low and high expression (arrows). Scale bar: $100 \mu \mathrm{m}$. Insets: 2x magnification (a). Graph shows that $D D R 2$ expression is related to CD68 levels $(p=0.0001)(b) . D D R 2$ and CD68 status positive correlation in breast IDC ( $R=0.32$; $p<2.2 e-16)$ and ILC $(R=0.46 ; p<3.3 e-07)$ samples obtained from METABRIC BC Datasets $(c, d)$. 
with a high risk of relapse, due to the ineffectiveness of hormone and HER-2 therapies, like in triple-negative subtype, as well as the development of chemoresistance. The observed potential connection between DDR2 and the TME markers could put some light on the treatment options for these patients, focusing on the combination of DDR2 inhibitors and stromal-directed drug regimens with the present clinical approaches.

\section{Data Availability}

The data used to support the findings of this study are available upon request from Dr. Beatriz Arteta through the email address beatriz.arteta@ehu.eus.

\section{Conflicts of Interest}

The authors declare that they have no conflicts of interest.

\section{Acknowledgments}

The authors thank Basurto University Hospital (Bilbao, Spain) for supporting this study by the OSI grant.

\section{Supplementary Materials}

Table S1: genes positively correlated with DDR2 in invasive BC (TOP 100). PCC: Pearson Correlation Coefficient. (Supplementary Materials)

\section{References}

[1] N. Harbeck, F. Penault-Llorca, J. Cortes et al., "Breast cancer," Nature Reviews Disease Primers, vol. 5, no. 1, p. 66, 2019.

[2] F. Bray, J. Ferlay, I. Soerjomataram, R. L. Siegel, L. A. Torre, and A. Jemal, "Global cancer statistics 2018: GLOBOCAN estimates of incidence and mortality worldwide for 36 cancers in 185 countries," CA: A Cancer Journal for Clinicians, vol. 68, no. 6, pp. 394-424, 2018.

[3] G. Turashvili, J. Bouchal, G. Burkadze, and Z. Koláŕ, "Differentiation of tumours of ductal and lobular origin: I. Proteomics of invasive ductal and lobular breast carcinomas," Biomedical Papers, vol. 149, no. 1, pp. 57-62, 2005.

[4] R. Barroso-Sousa and O. Metzger-Filho, "Differences between invasive lobular and invasive ductal carcinoma of the breast: results and therapeutic implications," Therapeutic Advances in Medical Oncology, vol. 8, no. 4, pp. 261-266, 2016.

[5] N. Harbeck and M. Gnant, "Breast cancer," Lancet, vol. 389, no. 10074, pp. 1134-1150, 2017.

[6] E. Dalmau, A. Armengol-Alonso, M. Muñoz, and M. Á. Seguí-Palmer, "Current status of hormone therapy in patients with hormone receptor positive $(\mathrm{HR}+)$ advanced breast cancer," The Breast, vol. 23, no. 6, pp. 710-720, 2014.

[7] M. C. Figueroa-Magalhães, D. Jelovac, R. M. Connolly, and A. C. Wolff, "Treatment of HER2-positive breast cancer," Breast, vol. 23, no. 2, pp. 128-136, 2014.

[8] L. Yin, J.-J. Duan, X.-W. Bian, and S.-C. Yu, "Triple-negative breast cancer molecular subtyping and treatment progress," Breast Cancer Research, vol. 22, no. 1, p. 61, 2020.

[9] EBCTCG, C. Davies, J. Godwin et al., "Relevance of breast cancer hormone receptors and other factors to the efficacy of adjuvant tamoxifen: patient-level meta-analysis of randomised trials," Lancet, vol. 378, no. 9793, pp. 771-784, 2011.
[10] L. Yaghjyan, G. A. Colditz, L. C. Collins et al., "Mammographic breast density and subsequent risk of breast cancer in postmenopausal women according to tumor characteristics," Journal of the National Cancer Institute, vol. 103, no. 15, pp. 1179-1189, 2011.

[11] J. A. Tice, E. S. O’Meara, D. L. Weaver, C. Vachon, R. BallardBarbash, and K. Kerlikowske, "Benign breast disease, mammographic breast density, and the risk of breast cancer," Journal of the National Cancer Institute, vol. 105, no. 14, pp. 1043-1049, 2013.

[12] S. Kaushik, M. W. Pickup, and V. M. Weaver, "From transformation to metastasis: deconstructing the extracellular matrix in breast cancer," Cancer and Metastasis Reviews, vol. 35, no. 4, pp. 655-667, 2016.

[13] C. Walker, E. Mojares, and A. del Río Hernández, "Role of extracellular matrix in development and cancer progression," International Journal of Molecular Sciences, vol. 19, no. 10, p. 3028, 2018.

[14] G. Sökeland and U. Schumacher, "The functional role of integrins during intra- and extravasation within the metastatic cascade," Molecular Cancer, vol. 18, no. 1, p. 12, 2019.

[15] A. Shrivastava, C. Radziejewski, E. Campbell et al., “An orphan receptor tyrosine kinase family whose members serve as nonintegrin collagen receptors," Molecular Cell, vol. 1, no. 1, pp. 25-34, 1997.

[16] R. R. Valiathan, M. Marco, B. Leitinger, C. G. Kleer, and R. Fridman, "Discoidin domain receptor tyrosine kinases: new players in cancer progression," Cancer and Metastasis Reviews, vol. 31, no. 1-2, pp. 295-321, 2012.

[17] W. Jing, L. Li, X. Zhang et al., "Genetic profiling of breast cancer with and without preexisting metabolic disease," Translational Oncology, vol. 13, no. 2, pp. 245-253, 2020.

[18] T. Ren, J. Zhang, J. Zhang, X. Liu, and L. Yao, "Increased expression of discoidin domain receptor 2 (DDR2): a novel independent prognostic marker of worse outcome in breast cancer patients," Medical Oncology, vol. 30, no. 1, p. 397, 2013.

[19] K. Zhang, C. A. Corsa, S. M. Ponik et al., "The collagen receptor discoidin domain receptor 2 stabilizes SNAIL1 to facilitate breast cancer metastasis," Nature Cell Biology, vol. 15, no. 6, pp. 677-687, 2013.

[20] E. Slocum and D. Germain, "Collagen and PAPP-A in the etiology of postpartum breast cancer," Hormones and Cancer, vol. 10, no. 4-6, pp. 137-144, 2019.

[21] S. V. Bayer, W. R. Grither, A. Brenot et al., "DDR2 controls breast tumor stiffness and metastasis by regulating integrin mediated mechanotransduction in CAFs," Elife, vol. 8, Article ID e45508, 2019.

[22] C. A. S. Corsa, A. Brenot, W. R. Grither et al., "The action of discoidin domain receptor 2 in basal tumor cells and stromal cancer-associated fibroblasts is critical for breast cancer metastasis," Cell Reports, vol. 15, no. 11, pp. 2510-2523, 2016.

[23] C. Zeltz, I. Primac, P. Erusappan, J. Alam, A. Noel, and D. Gullberg, "Cancer-associated fibroblasts in desmoplastic tumors: emerging role of integrins," Seminars in Cancer Biology, vol. 62, pp. 166-181, 2020.

[24] K. Shiga, M. Hara, T. Nagasaki, T. Sato, H. Takahashi, and H. Takeyama, "Cancer-associated fibroblasts: their characteristics and their roles in tumor growth," Cancers, vol. 7, no. 4, pp. 2443-2458, 2015.

[25] S. Salimifard, A. Masjedi, M. Hojjat-Farsangi et al., "Cancer associated fibroblasts as novel promising therapeutic targets in breast cancer," Pathology, Research \& Practice, vol. 216, no. 5, Article ID 152915, 2020. 
[26] E. Shirmohammadi, S.-E. S. Ebrahimi, A. Farshchi, and M. Salimi, "The efficacy of etanercept as anti-breast cancer treatment is attenuated by residing macrophages," $B M C$ Cancer, vol. 20, no. 1, p. 836, 2020.

[27] D. Aran, R. Camarda, J. Odegaard et al., "Comprehensive analysis of normal adjacent to tumor transcriptomes," Nature Communications, vol. 8, no. 1, p. 1077, 2017.

[28] K. A. Toy, R. R. Valiathan, F. Núñez et al., "Tyrosine kinase discoidin domain receptors DDR1 and DDR2 are coordinately deregulated in triple-negative breast cancer," Breast Cancer Research and Treatment, vol. 150, no. 1, pp. 9-18, 2015.

[29] B. Pereira, S.-F. Chin, O. M. Rueda et al., "The somatic mutation profiles of 2,433 breast cancers refine their genomic and transcriptomic landscapes," Nature Communications, vol. 7, no. 1, Article ID 11479, 2016.

[30] C. Curtis, S. P. Shah, S. P. Shah et al., "The genomic and transcriptomic architecture of 2,000 breast tumours reveals novel subgroups," Nature, vol. 486, no. 7403, pp. 346-352, 2012.

[31] J. Gao, B. A. Aksoy, U. Dogrusoz et al., "Integrative analysis of complex cancer genomics and clinical profiles using the cBioPortal," Science Signaling, vol. 6, no. 269, p. pl1, 2013.

[32] E. Cerami, J. Gao, U. Dogrusoz et al., "The cBio cancer genomics portal: an open platform for exploring multidimensional cancer genomics data: figure 1," Cancer Discovery, vol. 2, no. 5, pp. 401-404, 2012.

[33] Y. Chen, Y. Huang, X. Gao et al., "CCND1 amplification contributes to immunosuppression and is associated with a poor prognosis to immune checkpoint inhibitors in solid tumors," Frontiers in Immunology, vol. 11, p. 1620, 2020.

[34] A. Lundberg, L. S. Lindström, J. Li et al., "The long-term prognostic and predictive capacity of cyclin D1 gene amplification in 2305 breast tumours," Breast Cancer Research, vol. 21 , no. 1, p. 34, 2019.

[35] D. S. Chandrashekar, B. Bashel, S. A. H. Balasubramanya et al., "UALCAN: a portal for facilitating tumor subgroup gene expression and survival analyses," Neoplasia, vol. 19, no. 8, pp. 649-658, 2017.

[36] Z. Tang, C. Li, B. Kang, G. Gao, C. Li, and Z. Zhang, "GEPIA: a web server for cancer and normal gene expression profiling and interactive analyses," Nucleic Acids Research, vol. 45, no. W1, pp. W98-W102, 2017.

[37] M. V. Kuleshov, M. R. Jones, A. D. Rouillard et al., "Enrichr: a comprehensive gene set enrichment analysis web server 2016 update," Nucleic Acids Research, vol. 44, no. W1, pp. W90-W97, 2016.

[38] J. Liu, J. X. Shen, H. T. Wu et al., "Collagen 1A1 (COL1A1) promotes metastasis of breast cancer and is a potential therapeutic target," Discovery Medicine, vol. 25, pp. 211-223, 2018.

[39] J. M. Szulczewski, D. R. Inman, M. Proestaki, J. Notbohm, B. M. Burkel, and S. M. Ponik, "Directional cues in the tumor microenvironment due to cell contraction against aligned collagen fibers," Acta Biomater, vol. 129, 2021.

[40] K. Kesh, V. K. Gupta, B. Durden et al., "Therapy resistance, cancer stem cells and ECM in cancer: the matrix reloaded," Cancers, vol. 12, no. 10, p. 3067, 2020.

[41] B. L. Sprague, P. M. Vacek, S. E. Mulrow et al., "Collagen organization in relation to ductal carcinoma in situ pathology and outcomes," Cancer Epidemiol Biomarkers and Prevention, vol. 30, pp. 80-88, 2021.

[42] E. Slocum, A. Craig, A. Villanueva, and D. Germain, "Parity predisposes breasts to the oncogenic action of PAPP-A and activation of the collagen receptor DDR2," Breast Cancer Research, vol. 21, no. 1, p. 56, 2019.
[43] B. Arneth, "Tumor microenvironment," Medicina, vol. 56, no. 1, p. 15, 2019.

[44] S. Morikawa, P. Baluk, T. Kaidoh, A. Haskell, R. K. Jain, and D. M. McDonald, "Abnormalities in pericytes on blood vessels and endothelial sprouts in tumors," American Journal Of Pathology, vol. 160, no. 3, pp. 985-1000, 2002.

[45] B. Gok Yavuz, G. Gunaydin, M. E. Gedik et al., "Cancer associated fibroblasts sculpt tumour microenvironment by recruiting monocytes and inducing immunosuppressive PD1+ TAMs," Scientific Reports, vol. 9, no. 1, p. 3172, 2019.

[46] Q. Li, M. Li, K. Zheng, S. Tang, and S. Ma, "Expression pattern analysis and drug differential sensitivity of cancer-associated fibroblasts in triple-negative breast cancer," Translational Oncology, vol. 14, no. 1, Article ID 100891, 2021.

[47] B. Poudel, Y.-M. Lee, and D.-K. Kim, "DDR2 inhibition reduces migration and invasion of murine metastatic melanoma cells by suppressing MMP2/9 expression through ERK/ NF- B pathway," Acta Biochimica et Biophysica Sinica, vol. 47, no. 4, pp. 292-298, 2015.

[48] E. S. Radisky and D. C. Radisky, "Matrix metalloproteinases as drivers and therapeutic targets in breast cancer," Frontiers in Bioscience, vol. 20, no. 7, pp. 1144-1163, 2015.

[49] R. Kalluri, "The biology and function of fibroblasts in cancer," Nature Reviews Cancer, vol. 16, no. 9, pp. 582-598, 2016.

[50] K. A. Gieniec, L. M. Butler, D. L. Worthley, and S. L. Woods, "Cancer-associated fibroblasts-heroes or villains?" British Journal of Cancer, vol. 121, no. 4, pp. 293-302, 2019.

[51] A. M. Malfitano, S. Pisanti, F. Napolitano, S. Di Somma, R. Martinelli, and G. Portella, "Tumor-associated macrophage status in cancer treatment," Cancers, vol. 12, no. 7, p. 1987, 2020.

[52] Y. Pan, Y. Yu, X. Wang, and T. Zhang, "Tumor-associated macrophages in tumor immunity," Frontiers in Immunology, vol. 11, Article ID 583084, 2020.

[53] Z. Yan, S. Jin, Z. Wei et al., "Discoidin domain receptor 2 facilitates prostate cancer bone metastasis via regulating parathyroid hormone-related protein," Biochimica et Biophysica Acta - Molecular Basis of Disease, vol. 1842, no. 9, pp. 1350-1363, 2014.

[54] Y. Liu, Y. Li, N. Li et al., “TGF- $\beta 1$ promotes scar fibroblasts proliferation and transdifferentiation via up-regulating MicroRNA-21," Scientific Reports, vol. 6, no. 1, Article ID 32231, 2016.

[55] L. Caja, F. Dituri, S. Mancarella et al., "TGF- $\beta$ and the tissue microenvironment: relevance in fibrosis and cancer," International Journal of Molecular Sciences, vol. 19, no. 5, p. 1294, 2018.

[56] F. N. Soki, S. I. Park, and L. K. McCauley, "The multifaceted actions of PTHrP in skeletal metastasis," Future Oncology, vol. 8, no. 7, pp. 803-817, 2012.

[57] M. Iwano, D. Plieth, T. M. Danoff, C. Xue, H. Okada, and E. G. Neilson, "Evidence that fibroblasts derive from epithelium during tissue fibrosis," Journal of Clinical Investigation, vol. 110, no. 3, pp. 341-350, 2002.

[58] M. Zeisberg, C. Yang, M. Martino et al., "Fibroblasts derive from hepatocytes in Liver fibrosis via epithelial to mesenchymal transition," Journal of Biological Chemistry, vol. 282, no. 32, pp. 23337-23347, 2007. 